\title{
Splitting torsion theories over commutative rings
}

\section{John D. Fuelberth, James Kuzmanovich, and Thomas S. Shores}

The purpose of this paper is to completely characterize splitting torsion theories over commutative rings. In particular, if $(T, F)$ is a torsion theory for which $T(R)=0$, then $(T, F)$ is a splitting theory if and only if $T$ contains only a finite number of nonisomorphic simple modules and every module in $T$ is semisimple injective. In addition, an ideal theoretic characterization of splitting torsion theories is given, of which one consequence is that splitting torsion theories are TTF; furthermore, if $R$ is also noetherian, then such torsion theories are centrally splitting. The known theorems concerning the splitting of the Goldie and simple torsion theories (for commutative rings) are derived from our theorem.

In this paper, all rings are commutative with identity and all modules are unitary. The category of all $R$-modules will be denoted by $M_{R}$.

A torsion theory for $M_{R}$ is a pair $(T, F)$ of classes of $R$-modules satisfying:

(i) $T \cap F=\{0\}$;

(ii) $T$ is closed under homomorphic images;

(iii) $F$ is closed under submodules;

Received 25 September 1975. The third author was supported by a grant from the University of iNebraska Research Council. 
(iv) for each $A \in M_{R}$, there is a submodule $T(A) \in T$ called the torsion submodule for which $A / T(A) \in F$.

$T$ is the torsion class and $F$ is the torsionfree class. A torsion theory $(T, F)$ is called hereditary provided that $T$ is closed under submodules. A hereditary torsion theory is uniquely associated with an idempotent topologizing fizter of right ideals $F(T)=\{I: R / I \in T\}$. In this paper, all torsion theories will be hereditary. The torsion theory $(T, F)$ is called TTF if $F(T)$ has a minimal element. A torsion theory is said to be a splitting torsion theory provided $T(A)$ is a direct summand of $A$ for all $R$-modules $A$. A torsion theory $(T, F)$ is said to be of simple type provided that every nonzero submodule of every homomorphic image of a module in $T$ has nonzero socle. The torsion theory of simple type for which every simple module is torsion is called the simple torsion theory.

The following lemma is used to prove a "finiteness" condition.

LEMMA 1. Let $Y=\left\{M_{i}: i \in I\right\}$ be a set of distinet maximal ideals of $R$ which are also minimal prime ideals of $R$. If $\oplus\left\{R / M_{i}: i \in I\right\}$ is a direct summand of $\prod\left\{R / M_{i}: i \in I\right\}$, then $Y$ is a finite set.

Proof. The Zariski topology on the spectrum of $R$ induces a topology on $Y$. First we will show that $Y$ is noetherian; that is, $Y$ does not have an infinite strictly decreasing sequence of closed sets. For if $V_{1} \supsetneqq V_{2} \supsetneqq \cdots$ is such a sequence, let $M_{n} \in V_{n} V_{n+1}$. Set $Y^{\prime}=\left\{M_{n}: n \geq 1\right\}, \quad V_{n}^{\prime}=V_{n} \cap Y^{\prime}$. Note that $\left\{V_{n}^{\prime}\right\}$ is a strictly decreasing sequence of closed subsets of $y^{\prime}$ since $M_{n} \in V_{n}^{\prime} \backslash V_{n+1}^{\prime}$. Since $\oplus\left\{R / M_{i}: i \in I\right\}$ is a direct summand of $\prod\left\{R / M_{i}: i \in I\right\}$, $\oplus\left\{R / M_{n}: n \geq 1\right\}$ is a direct summand of $\prod\left\{R / M_{n}: n \geq 1\right\}$. Let $x=\left(x_{n}\right)$ be an element of $\prod\left\{R / M_{n}\right\}$ such that $x_{n} \neq 0$ for each $n$. For each $n$, define $P_{n}(x)=n\left\{\operatorname{ann}_{R} x_{k}: k \geq n\right\}$. Since $\oplus\left\{R / M_{n}\right\}$ is a direct summand of $\left\lceil\left\lceil\left\{R / M_{n}\right\}\right.\right.$, it follows from a result of Sarath and Varadarajan [7, Theorem 1, p. 521] that the family of ideals $\left\{P_{n}(x)\right\}$ must be finite. Hence, there is a $k$ such that $P_{k}(x)=P_{k+1}(x)$. Then 
$V\left(P_{k}(x)\right)=V\left(P_{k+1}(x)\right)$ (where $V(I)$ denotes the closed set determined by the ideal $I$ in the Zariski topology). Hence

$$
V_{k}^{\prime}=V\left(P_{k}(x)\right) \cap Y^{\prime}=V\left(P_{k+1}(x)\right) \cap Y^{\prime}=V_{k+1}^{\prime},
$$

a contradiction. Hence, $y$ is noetherian.

It is well known that the set of minimal primes of $R$ is a Hausdorff space relative to the Zariski topology [3]. Suppose $Y$ is infinite.

Since any infinite Hausdorff space contains an infinite discrete subspace, $Y$ contains an infinite discrete subspace $X$. Since $Y$ is noetherian, this is impossible. Hence, $Y$ is finite. //

An interesting application of Lemma $I$ is the following generalization of a result of Levine [4, Proposition 2,4].

COROLLARY 2. Let $\left\{S_{i}: i \in I\right\}$ be a family of nonisomorphic injective simple modules over a commutative ring such that $\oplus\left\{S_{i}: i \in I\right\}$ is injective. Then $|I|$ is finite.

Proof. Suppose that $S_{i} \simeq R / M_{i}$ for suitable distinct maximal ideals $M_{i}, \quad i \in I$, of $R$. Then the localizations $R_{M_{i}}$ are fields by [5,

Lemma 8]. Since localization at $M_{i}$ preserves the primes contained in $M_{i}$, we have that each $M_{i}$ is a minimal prime of $R$. However, $\Theta\left\{R / M_{i}: i \in I\right\}$ is certainly a summand of $\prod\left\{R / M_{i}: i \in I\right\}$, whence the result follows from Lemma 1 . //

The following result is due to Goodearl.

LEMMA 3. Let $(T, F)$ be a splitting theory for $M_{R}$ and let $T(R)=0$. If $H \in F(T)$, then $R / H$ is a perfect ring and hence $T$ is of simple type.

Proof. The proof is essentially that of [2, Theorem 5.3]. //

The next lemma is due to Teply and Fuelberth [9, Lemma 4.3 and Lemma 4.5] and we include it here for completeness.

LEMMA 4. Let $(T, F)$ be a splitting torsion theory of simple type for $M_{R}$ such that $T(R)=0$. If $M \in F(T)$ is a maximal ideal of $R$, 
then $R_{M}$ is a principal ideal domain and the simple theory for $M_{R_{M}}$ is a splitting theory.

If $I$ is an ideal of $R, A$ an $R$-module, then the $I$-socle of $A$ is $\operatorname{soc}(A, I)=\{x \in A: x I=0\}$.

We now state our main result.

THEOREM 5. Let $e$ be an idempotent of $R$ and $Y$ a finite set of maximal ideals of $R$ containing $e$ such that $R_{M}$ is a fiezd for all $M \in Y$. For each R-module A define

$$
T(A)=A e+\left(\sum\{\operatorname{soc}(A, M): M \in Y\}\right) .
$$

Then the sum is direct, $T$ is a splitting torsion theory for $R$ and every splitting theory for $R$ is so obtained. Note that $T(R)=$ Re in this case.

Proof. The directness of the sums of the fomula for $T(A)$ is routine. In order to show that the formula gives a splitting theory, it suffices to treat the case $T(A)=\operatorname{soc}(A, M)$ since we may then split off the component submodules one at a time to obtain the desired result. Since $R_{M}$ is a field, $R / M$ is an injective $R$-module by [5, Lemma 8]. Moreover, observe that for any index set $I, \Theta\{R / M: i \in I\}$ is a direct summand of $T T\{R / M: i \in I\}$ by vector space theory. Thus $\oplus R / M$ is an injective $R$-module and $T$ is a splitting torsion theory.

Conversely suppose that $(T, F)$ is a splitting torsion theory. Then $T(R)=R e$ where $e^{2}=e$ and $(T, F)$ induces a splitting theory $\left(T^{\prime}, F^{\prime}\right)$ for $R^{\prime}=R(1-e)$ for which $T^{\prime}\left(R^{\prime}\right)=0$. Since any $A \in M_{R}$ decomposes as $A=e A+(1-e) A$, we may assume that $T(R)=0$ and $e=0$.

By Lemma 3, $(T, F)$ is of simple type. If $M \in F(T)$ is a maximal ideal of $R, R_{M}$ is a principal ideal domain and the simple theory for $M_{R_{M}}$ is splitting by Lemma 4. $R_{M}$ is then a field. For if not, since $R_{M}$ is a principal ideal domain, the simple theory for $R_{M}$ is the classical torsion theory for an integral domain. But any integral domain for which the classical torsion theory splits is a field [6], a contradiction. 
We now show that

$\left\{M_{i}: M_{i} \in F(T)\right.$ and $M_{i}$ is a maximal ideal of $R$ for all $\left.i \in I\right\}$ is a finite set. Consider $X=\prod\left\{R / M_{i}: i \in I\right\}$. If $x=\left(x_{i}\right) \in T(X)$, then $\operatorname{ann}_{R} x=\cap\left\{M_{i}: x_{i} \neq\right.$ of $\in F(T)$. By Lemma $3, R / a n n_{R} x$ is a perfect ring so $x_{i} \neq 0$ for at most a finite number of $i \in I$. Hence $T(X)=\oplus\left\{R / M_{i}: i \in I\right\}$. By a previous paragraph, $R_{M_{i}}$ is a field for all $i \in I$; hence $R / M_{i}$ is $R$-injective by [5, Lemma 8]. Since $R \rightarrow R_{M}$ preserves primes contained in $M$, it follows that $M$ is a minimal prime as well. Since $T(X)$ is a summand of $X$, by Lemma $I, I$ is a finite set.

Let $J=\cap\left\{M_{i}: i \in I\right\}$. Since $R_{M_{i}}$ is a field for all $i \in I$, $M_{i}^{2}=M_{i}$ for all $i$. It follows that $J$ is the product of all the $M_{i}$ for $i \in I$ and $J^{2}=J$. Since $R / K$ is a perfect ring for all $K \in F(T)$, it is routine to check that $J \subseteq K$ for all $K \in F(T)$. Hence $R / K$ is a module over the semisimple ring $R / J \simeq \oplus\left\{R / M_{i}: M_{i} \in I\right\}$ and $R / K$ is completely reducible. Hence, for any $A \in T$, $A=\Theta\left\{\operatorname{soc}\left(A, M_{i}\right): i \in I\right\}$. Thus the formula given in the theorem holds.//

REMARK. Note that if $T(R)=0$, "Theorem 5 states that $(T, F)$ is a splitting theory if and only if every element of $T$ is semisimple (hence injective) and $T$ contains only a finite number of nonisomorphic simple $R$-modules.

COROLLARY 6. If (T, F) is a splitting torsion theory over a commutative ming, then $T$ is a TTF class.

A torsion theory $(T, F)$ is said to be central splitting provided $R=e R \uplus f R$ for central idempotents $e$ and $f$ of $R$ and

$$
T=\{M: M f R=0\}, F=\{M: M e R=0\} .
$$

Central splitting torsion theories for indecomposable rings are trivial.

The following corollary characterizes splitting torsion theories for commutative noetherian rings. 
COROLLARY 7. Every splitting torsion theory over a commitative noetherian ring is centrally splitting.

Proof. If $(T, F)$ is splitting, then any maximal ideal $M \in F(T)$ is idempotent by the proof of Theorem 5 . Since $M$ is finitely generated, $M=e R$ for an idempotent $e$ of $R$. Since $\operatorname{soc}(A, M)=A(1-e),(T, F)$ is centrally splitting. //

The above results show that splitting but not centrally splitting torsion theories for commutative regular rings are plentiful. For example, the maximal quotient ring $Q$ of $C[0,1]$ is a self injective regular ring with zero socle. The theorem shows that $Q$ has many splitting torsion theories.

A ring $R$ is called semiartinian if every $R$-module has a nonzero socle. Let $(S, F)$ denote the simple torsion theory. The following result is due to Teply and Fuelberth [9, Theorem 5.1].

COROLLARY 8. (S, F) is a splitting torsion theory for a commutative ring $R$ if and only if $R$ is semiartinain.

Proof. Let $(S, F)$ be a splitting torsion theory for $R$ and assume that $S(R)=0$. Then every simple module is injective (see the remark following Theorem 5); hence $R$ is a regular ring by [5, Theorem 6]. By Theorem 5, $R$ has only finitely many maximal ideals so $R$ is semisimple artinian, a contradiction to $S(R)=0$. / /

One well known torsion theory is the Goldie theory denoted by $(G, N)$. $(G, N)$ is the hereditary torsion theory for which $F(G)$ is the smallest idempotent topologizing filter containing all essential ideals of $R$. $N$ is the class of all nonsingular modules. If $G(R)=0$, then $G(M)$ is the singular submodule of $M$ for all modules $M$.

The following Corollary is a new proof of a theorem due to Cateforis and Sandomierski [1, Theorem 2.1].

COROLLARY 9. (G,N) is a splitting theory for $M_{R}$ if and only if $R \simeq S \uplus T$ where $S$ is a ring with essential singular submodule and $T$ is a regular semiartinian ming such that $T / \operatorname{soc}(T)$ is a finite product of fields. In this case, if $S=e R$ and $Y$ is the (possibly empty and necessarily finite) set of maximal ideals containing $S \uplus \operatorname{soc}(T)$, then for any R-module $A$, 


$$
G(A)=A e \uplus(\oplus\{\operatorname{soc}(A, M): M \in Y\}) .
$$

Proof. Suppose $(G, N)$ splits, so $R=S \oplus T$ where $G\left(S_{R}\right)=S=e R$ and $G\left(T_{R}\right)=0$. It follows that $S$ is a singular ring and $T$ is a nonsingular splitting ring. Therefore $S_{S}$ is an essential extension of its singular submodule and moreover, for an arbitrary $R$-module $A$, $G(A)=A e \otimes G_{T}(A(1-e))$. So it remains to compute $G_{T}$ where $G_{T}$ is the Goldie torsion theory for $M_{T}$. By Corollary $6, G_{T}$ is a TTF class so by [8, Theorem 1.3] every nonsingular T-module has nonzero socle. It follows that $T$ is an essential extension of $\operatorname{soc}(T)$. By Theorem 5, for any $B \in M_{T}$,

$$
G_{T}(B)=B f \oplus(\Theta\{\operatorname{soc}(B, M): M \in Y\})
$$

for a suitable collection $Y$ of maximal ideals $M$ of $T$ and idempotent $f \in T$. Since $G_{T}(T)=0, f=0$. Also, it follows that the only essential ideals of $T$ are intersections of subsets of $Y$. But every ideal containing $\operatorname{soc}(T)$ is essential and indeed these are the only essential ideals. Hence $T / \operatorname{soc}(T)$ is semisimple artinian and a finite product of fields.

CLAIM. $T$ is a semiprime ring. If $I$ is any ideal of $T$ for which $I^{2}=0$, then there must be a simple module $S \subset \operatorname{soc}(T)$ such that $S^{2}=0$. Let $S^{\prime}$ be any simple submodule of $\operatorname{soc}(T)$. If $S^{\prime} \simeq S$, then $S S^{\prime} \simeq S^{2}=0$. If $S^{\prime} \neq S$, then $S^{\prime} S=0$ since the $\operatorname{soc}(T)$ is a direct sum of its homogeneous components. Thus $S[\operatorname{soc}(T)]=0$ and $S \subset G_{T}(T)=0$, a contradiction.

Hence $T$ is semiprime and semiartinian and $T$ is regular.

Conversely, suppose $R=S \uplus T$ has the prescribed form, where $S=e R$. Then again for all $R$-modules $A$,

$$
G(A)=G_{S}(A) \uplus G_{T}(A)=e A \uplus G_{T}(A(1-e)) .
$$

The maximal essential ideals of $T$ are precisely the maximal ideals containing soc $(T)$. For each such maximal ideal $M, e R \leftrightarrow M$ is an essential maximal ideal of $R$. Let $Y$ be the finite set of such ideals. 
For each $N \in Y, R / N$ is $T$-injective and hence $R$-injective. It follows easily that for all $R$-modules $A$,

$$
G(A)=A e \uplus(\Theta\{\operatorname{soc}(A, M): M \in Y\}) \text {. }
$$

Thus $(G, N)$ is a splitting torsion theory by Theorem 5 . //

\section{References}

[1] Vasily C. Cateforis and Francis L. Sandomierski, "The singular submodule splits off", J. Algebra 10 (1968), 149-165.

[2] K.R. Goodearl, Singular torsion and the splitting properties (Mem. Amer. Math. Soc. 124. Amer. Math. Soc., Providence, Rhode Island Island, 1972).

[3] M. Henriksen and M. Jerison, "The space of minimal prime ideals of a commutative ring", Trans. Amer. Math. Soc. 115 (1965), 110-130.

[4] Jeffrey Levine, "On the injective hulls of semisimple modules", Trans. Amer. Math. Soc. 155 (1971), 115-126.

[5] Alex Rosenberg and Daniel Zelinsky, "Finiteness of the injective hull", Math. 2. 70 (1958/59), 372-380.

[6] Joseph Rotman, "A characterization of fields among the integral domains", An. Acad. Brasiz. Ci. 32 (1960), 193-194.

[7] B. Sarath and K. Varadarajan, "Injectivity of direct sums", Comm. Algebra 1 (1974), 517-530.

[8] Mark L. Teply, "Some aspects of Goldie's torsion theory", Pacific J. Math. 29 (1969), 447-459.

[9] Mark L. Teply and John D. Fuelberth, "The torsion submodule splits off", Math. Ann. 188 (1970), 270-284.

Department of Mathematics,

University of Northern Colorado,

Greeley,

Colorado, USA;
Department of Mathematics, Wake Forest University, Winston-Salem, North Carolina, USA;

Department of Mathematics,

University of Nebraska,

Lincoln,

Nebraska, USA. 